\title{
The subgenual cingulate gyrus exhibits lower rates of bifurcation in schizophrenia than in controls, bipolar disorder and depression
}

\author{
Matthew R. Williams ${ }^{1,2 *}$, Ronald K. B. Pearce ${ }^{2}$, Steven R. Hirsch ${ }^{2}$, Olaf Ansorge ${ }^{3}$, Maria Thom ${ }^{4}$, \\ Michael Maier ${ }^{5}$ \\ ${ }^{1}$ King's College London, Institute of Psychiatry, De Crespigny Park, London, UK \\ ${ }^{2}$ Neuropathology Unit, Department of Clinical Neuroscience, Division of Neuroscience \& Mental Health, Imperial College London, \\ Charing Cross Hospital, London, UK \\ ${ }^{3}$ Department of Neuropathology, The Radcliffe Infirmary, Oxford, UK \\ ${ }^{4}$ Institute of Neurology, UCL, London, UK \\ ${ }^{5}$ Trust HQ, West London Mental Health NHS Trust, Middlesex, UK \\ Email: ${ }^{*}$ Matthew.r.williams@imperial.ac.uk
}

Received 23 August 2012; revised 25 September 2012; accepted 5 October 2012

\begin{abstract}
The subgenual cingulate cortex has been found to be different in structure and function in mood and affective disorders compared to healthy individuals. Imaging studies have shown a decrease in function of the subgenual region in bipolar disorder and depression, with overall glial number shown to be decreased in these disorders. Decreases in subgenual grey matter in SZ have been observed also. In this neuropathological study upon formalin-fixed coronal brain sections we describe the morphological finding of decreased frequency of subgenual cingulate crown bifurcation $(p=0.02)$ as compared to control, bipolar and depression cases. This suggests that the cingulate cortex in schizophrenia may be morphologically distinct in utero formation, potentially enabling an early identification of high-risk individuals.
\end{abstract}

Keywords: Neuropathology; Schizophrenia; Cortical; Flattening

\section{INTRODUCTION}

The subgenual cingulate cortex (SCC), part of Brodmann area $24 \mathrm{a}$, is a region of interest in schizophrenia (SZ) and bipolar disorder (BPD). The anterior cingulate cortex (ACC) lies on the medial surface of the cerebral hemisphere, covering the anterior part of the corpus callosum. It is defined as Broadmann area 24a. The ACC extends from the subgenual ventral terminus and continues rostal to the genu of the corpus callosum following the dorsal surface.

"Corresponding author.
The ACC has been implicated in regulation of emotional tional states, and in cognitive and attentional processes [1-5]. Projections travel directly from the SCC cortical grey matter to the corpus callosum. DTI studies have demonstrated decreased anisotropy in this cingulum bundle in patients with chronic SZ [6,7]. MRI imaging studies have shown a decrease in SCC grey matter volume with decreased thickness in first episode schizophrenic patients $[8,9]$ and in chronic disease and that the extent of thinning is related to illness duration [10-12] and a decrease in overall anterior cingulate volume [13]. Meta-analysis has suggested that the ACC in SZ has slightly decreased overall volume in SZ compared to controls, although there is significant heterogeneity in published articles [14]. Decreased ACC volume has also been observed in BPD in SCC cortical grey and white matter $[15,16]$. Smaller subgenual volumes have also been reported in unmedicated recurrent depressive disorder (RDD) [17] and decreased ACC volume has been shown to correlate inversely with good clinical outcome [18].

Whilst stereological examination shows no change in overall volume in frontal cortices in schizophrenia [19, 20], cortical thinning has been observed in area $24 \mathrm{a}$ in both SZ and BPD [15,21], and a decrease in the grey matter density in schizophrenia has also been reported in the orbitofrontal, cingulate and supramarginal cortices [22].

Some studies have suggested that in schizophrenia this thinning is more pronounced in the left side of the SCC $[23,24]$, and also a tendency to thicker SCC cortical grey matter in major depression. In addition, there has been reported a decrease anterior cingulate white matter integrity in schizophrenia $[25,26]$, suggesting a widespread 
issue with the morphology of anterior cingulate in these disorders.

In this study we have taken coronal sections through the SCC in cases of SZ, BPD and RDD and control cases to examine the prevalence of cortical bifurcation. The size of the bifurcation within a gyrus means it is not visible by imaging and therefore requires a microscopic methodology. As the extent of folding of the cortex increases its surface area within a given gyral volume, potentially reflecting the number of cortical neurons, this pattern of morphology may reflect a smaller cortical volume present from initial neurodevelopment of the cingulate.

\section{METHODS}

A cohort of cases obtained from the Corsellis brain collection was used in neuropathological investigation of the cingulate in a series of studies (Williams, 2012). Details of the diagnostic groups are shown in Table 1. A total of 68 age and post-mortem interval (PMI) matched cases were used, with 20 control cases with no psychiatric disorder (NPD), $12 \mathrm{SZ}$ cases, $16 \mathrm{BPD}$ cases and 20 cases of RDD.

All cases underwent full neuropsychiatric review and were subject to full neuropathological screening. Cases involving heavy alcohol or drug use and those exhibiting significant pathology such as neurodegenerative disease, cerebral vascular disease, ischemic brain damage, CNS infections and traumatic brain injury were excluded from the study. The majority of samples come from the county of Essex but a smaller number came from national referrals [27]. Medical notes were reviewed by a consultant psychiatrist and patients were selected on fulfilment of the ICD-10 criteria for SZ and MDD, for robustness of diagnosis the brains of the three diagnostic groups all suffered from chronic illness. Assessment for any neurodegenerative, neurovascular or infectious pathology, including Parkinson's disease, was undertaken by a consultant neuropathologist and affected patients excluded. Patients with any recorded history of alcohol or drug abuse were also excluded. In SZ selection the presence of first-rank symptoms was a necessity, and cases with onset younger than 20 and older than 30 were excluded.

Bilateral SCC and adjacent corpus callosum were dis- sected from formalin-fixed coronal blocks by a consultant neuropathologist and immersed in 10\% formalin (4\% formaldehyde v/v) until processing. Processing involved serial immersion in formalin, alcohols, methanol and xylene overnight. Tissue blocks were then embedded in paraffin wax and stored at $4^{\circ} \mathrm{C}$. Paraffin-embedded blocks were serially sectioned in the coronal plane at $10 \mu \mathrm{m}$ and mounted on $25 \times 75 \mathrm{~mm}$ electrostatic glass slides. Slides were blinded by an investigator not involved with the project before measurement. Sections from within 100 $\mu \mathrm{m}$ each case were stained with hematoxylin and eosin $(\mathrm{H} \& \mathrm{E})$ and cresyl-violet (CV) for accurate examination of the anatomy of the structures. Sections for $\mathrm{H} \& \mathrm{E}$ stain were submerged in xylene for 30 minutes and serially placed in troughs of $2 \times 100 \%, 90 \%, 70 \%$ ethanol (EtOH) and pure distilled water, each for 2 minutes. Slides were immersed in Mayer's haematoxylin stain for 5 minutes and subsequently washed in distilled water. Specimens were placed in differentiating agent acid-alcohol $(1 \%$ $\mathrm{HCl} / 70 \% \mathrm{EtOH}$ ) for 10 seconds and into distilled water for 2 minutes. Sections were then immersed in $1 \%$ eosin stain for 5 minutes and washed in distilled water. Finally, specimens were dehydrated in serial baths of $70 \%, 90 \%$ and $2 \times 100 \%$ IMS for 2 minutes each and submerged in xylene for 20 minutes before cover slips were fixed using DPX. Sections for CV stain were immersed in xylene for 30 minutes, incubated in $100 \%, 90 \%$ and then $70 \%$ alcohol for 10 minutes each, before immersion in ultrapure water. Sections were immersed in cresyl-violet stain for 5 minutes before washing in ultra-pure water and differentiation in $95 \%$ alcohol/ethanoic acid. After washing in ultra-pure water sections were dehydrated in serial alcohols, immersed in xylene and mounted with DPX.

Images of both $\mathrm{H} \& \mathrm{E}$ and $\mathrm{CV}$ stained coronal sections were taken using an Olympus microscope at $40 \times$ total magnification and captured at $2096 \times 1536$ resolution covering $2200 \times 1600 \mu \mathrm{m}$ area. Image analysis was performed using Image Pro Plus software (Media Cybernetics, US), calibrated using an optical graticule. In total for regions were measured in each case, both left and right $\mathrm{SCC}$ in $\mathrm{H} \& \mathrm{E}$ and $\mathrm{CV}$ sections. $\mathrm{H} \& \mathrm{E}$ sections were consulted to define the limits of the SCC grey matter.

Measures were taken in $\mathrm{CV}$ and $\mathrm{H} \& \mathrm{E}$ stained sections

Table 1. Summary group data. NPD—No Psychiatric Disorder; SZ—Schizophrenia; BPD—Bipolar Disorder; RDD—Recurrent Depressive Disorder. Age, PM delay and fixation time shown as means with SEM in brackets.

\begin{tabular}{cccccc}
\hline Diagnosis & $\mathbf{n}$ & Age/y & Sex ratio M/F & PM Delay/h & Fixation time/y \\
\hline NPD & 20 & $65.5(2.34)$ & $12 / 8$ & $44.2(7.38)$ & $10.2(0.52)$ \\
SZ & 12 & $58(6.44)$ & $6 / 6$ & $47.8(10.5)$ & $11.1(1.30)$ \\
BPD & 16 & $56.1(5.21)$ & $7 / 9$ & $50.5(7.46)$ & $19.4(2.02)$ \\
RDD & 20 & $47.6(3.12)$ & $6 / 14$ & $37.3(5.62)$ & $10.4(0.88)$ \\
\hline
\end{tabular}


by a scoring of incidence of bifurcation in the SCC from both hemispheres from each case, after comparable measures were taken from adjacent sulci and from the level of the crown. Bifurcation was defined as the existence of a sulcal-type projection into the crown of the primary SCC that were less than $50 \%$ the depth of both the callosal-cingulate sulcus and the primary cingulate sulcus, shown in Figure 1. Scoring of bifurcation of was taken from both left and right primary SCC in both CV and H \& E slides, totalling two measures per hemisphere and four measures per case. Bifurcation had to be present in both $\mathrm{CV}$ and $\mathrm{H} \& \mathrm{E}$ slides for inclusion.

Measures were performed blind to diagnosis and were unblinded by an investigator not involved with the project before analysis using Image Pro Plus. Multiple comparisons of confounding variables were performed using the general linear model univariate analysis using SPSS v16.0 statistical software (SPSS, USA). Direct analysis of the incidence of bifurcation was performed using Pearsons Chi-Square test (SPSS, USA).

\section{RESULTS}

The MDD group had a significantly lower age of death as compared to controls ( $\mathrm{p}=0.038,1$-way ANOVA). The MDD group $(n=20)$ contained 11 confirmed cases of suicide. The other disease groups also showed instances of suicide, which may contribute toward the non-significant trend downward in age in SZ (1 suicide, $\mathrm{n}=12$ ) and BPD (3 suicides, $\mathrm{n}=16$ ). The brain tissue of the BPD group was in formalin significantly longer than the other groups (Mean fixation time: NPD $11.3 \mathrm{yr}$ vs. BPD 19.7 $\mathrm{yr}, \mathrm{p}=0.0004$; NPD vs. SZ. $11.6 \mathrm{yr}, \mathrm{p}=0.074$; NPD vs. RDD. $11.8 \mathrm{yr}, \mathrm{p}=0.59$, ANOVA). There was no effect of sex, age, PM delay, incidence of suicide, fixation time or hemisphere on the incidence of bifurcation.

The incidence of bifurcation was 11/20 NPD cases (55\%), 2/12 SZ cases (17\%), 9/16 BPD cases (56\%) and 11/20 RDD cases (55\%), shown in Figure 2. The SZ group showed a significantly lower incidence of bifurcation than the controls or other diagnostic groups $(p=0.02$, Pearsons Chi-Square test, SPSS v16.0).

\section{DISCUSSION}

The results suggest that SCC bifurcation is less common in schizophrenia than in either controls, BPD or RDD. Whether this bifurcation is related to changed numbers of neuron or glial cells, or the function of the SCC, are unknown. Future neuropathological studies will be required to elicit further information of the cellular changes associated with altered cortical folding. Due to the nature of neuropathological studies it is not possible to measure total SCC volume using this type of study.

The cases of bipolar disorder had a longer mean PM

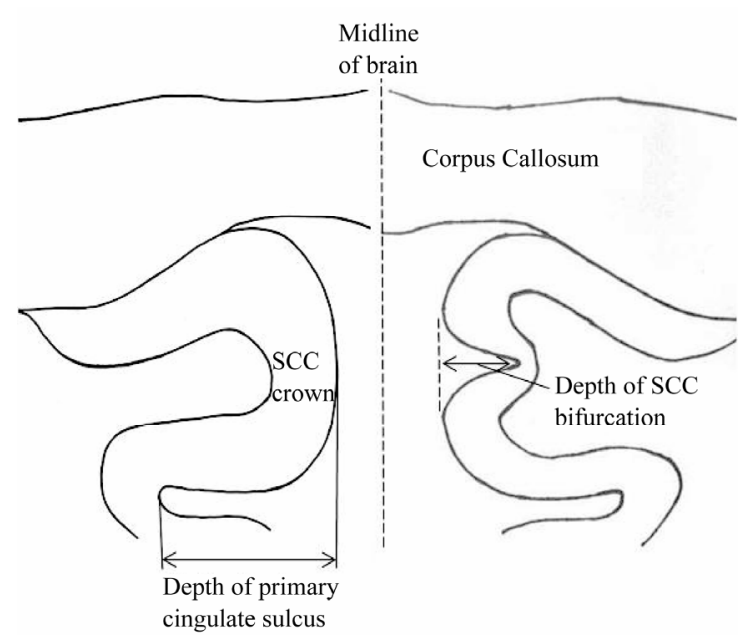

Figure 1. Schematic illustrating the measurement of the primary subgenual cingulate sulcus depth and a bifurcation in the SCC in the coronal plane.

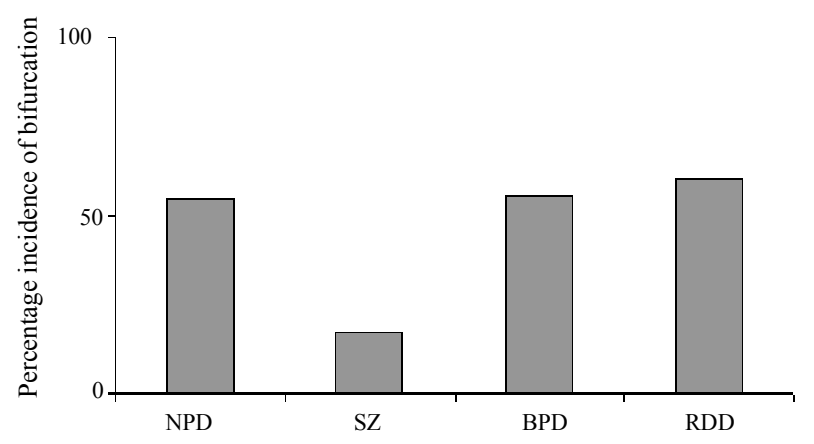

Figure 2. Incidence of bifurcation of the subgenual cingulate cortex as a percentage of cases. NPD-No Psychiatric Disorder; SZ-Schizophrenia; BPD—Bipolar Disorder; RDD—Recurrent Depressive Disorder.

delay than the cases from the other diagnostic groups. This was predominantly due to three male bipolar cases, numbers 32 (89 h), 34 (91 h) and 45 (100 h). The RDD group showed a reduced age of death, which may reflect a higher number of suicides amongst this group. Similarly male schizophrenia cases showed a younger age of death than female schizophrenia cases which was likely related to a higher number of suicides. Additionally the BPD cohort has a longer period of fixation, due to the lower number of BPD cases in the tissue bank, requiring tissue from earlier donations to be included. However these variables had no effect on the occurrence of bifurcation, or previous variables measured in the cingulate of these cases $[21,28]$. We did not have data on the illness duration. However as a requirement for inclusion was symptom onset between 20 - 30 yr this strongly correlated age, which had no effect on incidence of bifurcation.

Although the measures were collected from slides within $100 \mu \mathrm{m}$ of one another bifurcation was a gross 
anatomical occurrence and was easily visible with the naked eye on the blocks. Ideally the full volume of the SCC could be estimated by many serial sections, but this would require many blocks along the entire structure and this amount of tissue was not available. Also there would be errors involved due to the tissue shrinkage during fixation and processing. If these technical issues could be overcome then a volumetric study would be extremely useful as the measurements could be performed with far greater accuracy than in imaging.

Cortical folding changes in length and depth of temporal and frontal lobes have been reported in schizophrenia [29]. These changes been shown to be present before disease onset. Patients with adolescent onset schizophrenia have significantly more flattened curvature in the sulci and more steeped or peaked curvature in the gyri [30], and increased cortical folding in the superior frontal cortex and in gyral and sucal folding in temporal lobe of first episode schizophrenia [31,32]. High risk individuals have been observed to have altered cortical folding in the prefrontal cortex [33]. Examination of bifurcation may give additional information of changes in the cortex in schizophrenia between the scales of neuropathological reports of cell density and morphometry and the imaging data showing larger scale trends across cortical regions.

As gyral morphology is created in utero during the initial folding of the cortex, this suggests that morphological changes in schizophrenia may be hardwired early in life. If these can be identified then they may help with the early identification of high-risk individuals. Whilst large-scale changes in gyral folding may only be detectable in detailed analysis of the whole brain surface this study suggests that by looking at regions of high vulnerability signs of this change may be measurable at the microscopic level.

\section{ACKNOWLEDGEMENTS}

The authors are grateful to Prof. Federico Turkheimer for statistical advice. This was supported by funding from the Stanley and Starr foundations, and MRC-UK PET Methodology Programme Grant G1100809/1. This project was conducted under ethical permission granted by the London south west local ethics committee reference WL/02/12 (2002), and amendment WL/02/12/AM01, granted by the Ealing and WLMHT local research ethics committee (2006).

\section{REFERENCES}

[1] Bechara, A., Damasio, H., Tranel, D. and Damasio, A.R. (1997) Deciding advantageously before knowing the advantageous strategy. Science, 275, 1269-1272. doi:10.1126/science.275.5304.1293

[2] Bush, G., Vogt, B.A., Holmes, J., Dale, A.M., Greve, D. and Rosen, L.A. (2002) Dorsal anterior cingulate cortex: A role in reward-based decision making. PNAS USA, 99,

\section{3-528. doi:10.1073/pnas.012470999}

[3] Carter, C.S., MacDonald, A.M., Botvinick, M., Ross, L.L., Stenger, V.A., Noll, D. and Cohen, J.D. (2000) Parsing executive processes: Strategic vs. evaluative functions of the anterior cingulate cortex. PNAS USA, 97, 1944-1948. doi:10.1073/pnas.97.4.1944

[4] Carter, C.S., Mintun, M., Nichols, T. and Cohen, J.D. (1997) Anterior cingulate gyrus dysfunction and selective attention deficits in schizophrenia: $\left[{ }^{15} \mathrm{O}\right] \mathrm{H}_{2} \mathrm{O}$ PET study during single-trial Stroop task performance. American Journal of Psychiatry, 154, 1670-1675.

[5] Devinsky, O., Morrell, M.J. and Vogt, B.A. (1995) Contributions of anterior cingulate cortex to behaviour. Brain, 118, 279-306. doi:10.1093/brain/118.1.279

[6] Kubicki, M., Westin, C.F., Nestor, P.G., Wible, C.G., Frumin, M., Maier, S.E., Kikinis, R., Jolesz, F.A., McCarley, R.W. and Shenton, M.E. (2003) Cingulate fasciculus integrity disruption in schizophrenia: A magnetic resonance diffusion tensor imaging study. Biological Psychiatry, 54, 1171-1180. doi:10.1016/S0006-3223(03)00419-0

[7] Wang, F., Sun, Z., Cui, L., Du, X., Wang, X., Zhang, H., Cong, Z., Hong, N. and Zhang, D. (2004) Anterior cingulum abnormalities in male patients with schizophrenia determined through diffusion tensor imaging. American Journal of Psychiatry, 161, 573-575. doi:10.1176/appi.ajp.161.3.573

[8] Kasparek, T., Prikryl, R., Mikl, M., Schwarz, D., Ceskova, E. and Krupa, P. (2006) Prefrontal but not temporal grey matter changes in males with first-episode schizophrenia. Progress in Neuro-Psychopharmacology and Biological Psychiatry, 31, 151-157. doi:10.1016/j.pnpbp.2006.08.011

[9] Koo, M.S., Levitt, J.J., Salisbury, D.F., Nakamura, M., Shenton, M.E. and McCarley, R.W. (2008) A cross-sectional and longitudinal magnetic resonance imaging study of cingulate gyrus gray matter volume abnormalities in first-episode schizophrenia and first-episode affective psychosis. Archives of General Psychiatry, 65, 746-760. doi:10.1001/archpsyc.65.7.746

[10] Kuperberg, G.R., Broome, M.R., McGuire, P.K., David, A.S., Eddy, M., Ozawa, F., Goff, D., West, W.C., Williams, S.C., van der Kouwe, A.J., Salat, D.H., Dale, A.M. and Fischl, B. (2003) Regionally localized thinning of the cerebral cortex in schizophrenia. Archives of General Psychiatry, 60, 878-888. doi:10.1001/archpsyc.60.9.878

[11] Meisenzahl, E.M., Koutsouleris, N., Bottlender, R., Scheuerecker, J., Jäger, M., Teipel, S.J., Holzinger, S., Frodl, T., Preuss, U., Schmitt, G., Burgermeister, B., Reiser, M., Born, C. and Möller, H.J. (2008) Structural brain alterations at different stages of schizophrenia: A voxel-based morphometric study. Schizophrenia Research, 104, 44-60. doi:10.1016/j.schres.2008.06.023

[12] Narr, K.L., Bilder, R.M., Kim, S., Thompson, P.M., Szeszko, P., Robinson, D., Luders, E. and Toga, A.W. (2004) Abnormal gyral complexity in first-episode schizophrenia. Biological Psychiatry, 55, 859-867. doi:10.1016/j.biopsych.2003.12.027

[13] Fujiwara, H., Hirao, K., Namiki, C., Yamada, M., Shimizu, 
M., Fukuyama, H., Hayashi, T. and Murai, T. (2007) Anterior cingulate pathology and social cognition in schizophrenia: A study of gray matter, white matter and sulcal morphometry. Neuroimage, 36, 1236-1245. doi:10.1016/j.neuroimage.2007.03.068

[14] Baiano, M., David, A., Versace, A., Churchill, R., Balestrieri, M. and Brambilla, P. (2007) Anterior cingulate volumes in schizophrenia: A systematic review and a metaanalysis of MRI studies. Schizophrenia Research, 93, 1-12. doi:10.1016/j.schres.2007.02.012

[15] Bouras, C., Kovari, E., Hof, P.R., Riederer, B.M. and Giannakopoulos, P. (2001) Anterior cingulate cortex pathology in schizophrenia and bipolar disorder. Acta Neuropathologica, 102, 373-379.

[16] Nugent, A.C., Milham, M.P., Bain, E.E., Mah, L., Cannon, D.M., Marrett, S., Zarate, C.A., Pine, D.S., Price, J.L. and Drevets, W.C. (2006) Cortical abnormalities in bipolar disorder investigated with MRI and voxel-based morphometry. Neuroimage, 30, 485-497. doi:10.1016/j.neuroimage.2005.09.029

[17] Yucel, K., McKinnon, M.C., Chahal, R., Taylor, V.H., Macdonald, K., Joffe, R. and MacQueen, G.M. (2008) Anterior cingulate volumes in never-treated patients with major depressive disorder. Neuropsychopharmacology, 33, 3157-3163. doi:10.1038/npp.2008.40

[18] Frodl, T., Jäger, M., Born, C., Ritter, S., Kraft, E., Zetzsche, T., Bottlender, R., Leinsinger, G., Reiser, M., Möller, H.J. and Meisenzahl, E. (2008) Anterior cingulate cortex does not differ between patients with major depression and healthy controls, but relatively large anterior cingulate cortex predicts a good clinical course. Psychiatry Research, 163, 76-83.

doi:10.1016/j.pscychresns.2007.04.012

[19] Highley, J.R., Walker, M.A., Esiri, M.M., McDonald, B., Harrison, P.J. and Crow, T.J. (2001) Schizophrenia and the frontal lobes: Post-mortem stereological study of tissue volume. The British Journal of Psychiatry, 178, 337343. doi:10.1192/bjp.178.4.337

[20] Wright, I.C., Rabe-Hesketh, S., Woodruff, P.W., David, A.S., Murray, R.M. and Bullmore, E.T. (2000) Metaanalysis of regional brain volumes in schizophrenia. American Journal of Psychiatry, 157, 16-25.

[21] Williams, M.R., Chaudhry, R., Perera, S., Pearce, R.K.B., Hirsch, S.R., Ansorge, O., Thom, M. and Maier, M. (2012) Changes in cortical thickness in the frontal lobes in schizophrenia are a result of thinning of pyramidal cell layers. European Archives of Psychiatry and Clinical Neuroscience, May 19th Epub. http://www.ncbi.nlm.nih.gov/pubmed/22610045

[22] Heckers, S., Heinsen, H., Heinsen, Y.C. and Beckmann, H. (1990) Limbic structures and lateral ventricle in schizophrenia. A quantitative postmortem study. Archives of General Psychiatry, 47, 1016-1022. doi:10.1001/archpsyc. 1990.01810230032006

[23] Coryell, W., Nopoulos, P., Drevets, W., Wilson, T. and Andreasen, N.C. (2005) Subgenual prefrontal cortex volumes in major depressive disorder and schizophrenia: Diagnostic specificity and prognostic implications. Ame- rican Journal of Psychiatry, 162, 1706-1712. doi:10.1176/appi.ajp.162.9.1706

[24] Zetzsche, T., Preuss, U., Frodl, T., Watz, D., Schmitt, G., Koutsouleris, N., Born, C., Reiser, M., Möller, H.J. and Meisenzahl, E.M. (2007) In-vivo topography of structural alterations of the anterior cingulate in patients with schizophrenia: New findings and comparison with the literature. Schizophrenia Research, 96, 34-45. doi:10.1016/j.schres.2007.07.027

[25] Fujiwara, H., Namiki, C., Hirao, K., Miyata, J., Shimizu, M., Fukuyama, H., Sawamoto, N., Hayashi, T. and Murai, T. (2007) Anterior and posterior cingulum abnormalities and their association with psychopathology in schizophrenia: A diffusion tensor imaging study. Schizophrenia Research, 95, 215-222. doi:10.1016/j.schres.2007.05.044

[26] Sun, Z., Wang, F., Cui, L., Breeze, J., Du, X., Wang, X., Cong, Z., Zhang, H., Li, B., Hong, N. and Zhang, D. (2003) Abnormal anterior cingulum in patients with schizophrenia: A diffusion tensor imaging study. Neuroreport, 14, 1833-1836. doi:10.1097/00001756-200310060-00015

[27] Kasper, B.S., Taylor, D.C., Janz, D., Kasper, E.M., Maier, M., Williams, M.R. and Crow, T.J. (2010) Neuropathology of epilepsy and psychosis: The contributions of J. A. N. Corsellis. Brain, 133, 3795-3805. doi:10.1093/brain/awq235

[28] Williams, M.R., Hampton, T., Pearce, R.K.B., Hirsch, S.R., Ansorge, O., Thom, M. and Maier, M. (2012) Astrocyte decrease in the subgenual cingulate and callosal genu in schizophrenia. European Archives of Psychiatry and Clinical Neuroscience, June 4th Epub. http://www.ncbi.nlm.nih.gov/pubmed/22660922

[29] Narr, K.L., Thompson, P.M., Sharma, T., Moussai, J., Zoulman, C., Rayman, J. and Toga, A.W. (2001) Threedimensional mapping of gyral shape and cortical surface asymmetries in Schizophrenia: Gender Effects. American Journal of Psychiatry, 158, 244-255. doi:10.1176/appi.ajp.158.2.244

[30] White, T., Andreasen, N.C., Nopoulos, P. and Magnotta, V. (2003) Gyrification abnormalities in childhood- and adolescent-onset schizophrenia. Biological Psychiatry, 54, 418-426. doi:10.1016/S0006-3223(03)00065-9

[31] Narr, K.L., Toga, A.W., Szeszko, P., Thompson, P.M., Woods, R.P., Robinson, D., Sevy, S., Wang, Y., Schrock, K. and Bilder, R.M. (2005) Cortical thinning in cingulate and occipital cortices in first episode schizophrenia. Biological Psychiatry, 58, 32-40. doi:10.1016/j.biopsych.2005.03.043

[32] Harris, J.M., Yates, S., Miller, P., Best, J.J., Johnstone, E.C. and Lawrie, S.M. (2004) Gyrification in first-episode schizophrenia: A morphometric study. Biological Psychiatry, 55, 141-147. doi:10.1016/S0006-3223(03)00789-3

[33] Harris, J.M., Whalley, H., Yates, S., Miller, P., Johnstone, E.C. and Lawrie, S.M. (2004) Abnormal cortical folding in high-risk individuals: A predictor of the development of schizophrenia? Biological Psychiatry, 56, 182-189. doi:10.1016/j.biopsych.2004.04.007 